\title{
Large negative uniaxial magnetic anisotropy in highly distorted Co-ferrite thin films
}

Cite as: Appl. Phys. Lett. 114, 092408 (2019); https://doi.org/10.1063/1.5064845

Submitted: 09 October 2018 . Accepted: 07 February 2019. Published Online: 08 March 2019

Takeshi Tainosho, Jun-ichiro Inoue, Sonia Sharmin, Masaki Takeguchi, Eiji Kita, and Hideto Yanagihara (iD
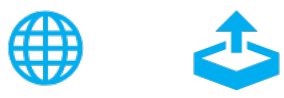

\section{ARTICLES YOU MAY BE INTERESTED IN}

Physicochemical origin of improvement of magnetic and transport properties of STT-MRAM cells using tungsten on FeCoB storage layer

Applied Physics Letters 114, 092407 (2019); https://doi.org/10.1063/1.5081912

Spin valve effect induced by spin-orbit torque switching

Applied Physics Letters 114, 092404 (2019); https://doi.org/10.1063/1.5086775

Giant non-volatile magnetoelectric effects via growth anisotropy in $\mathrm{Co}_{40} \mathrm{Fe}_{40} \mathrm{~B}_{20}$ films on PMN-PT substrates

Applied Physics Letters 114, 092401 (2019); https://doi.org/10.1063/1.5078787

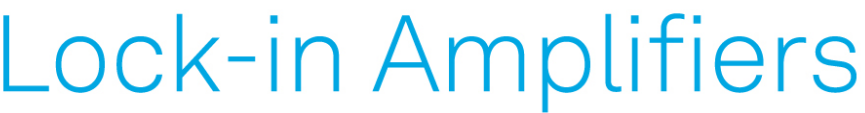

... and more, from DC to $600 \mathrm{MHz}$

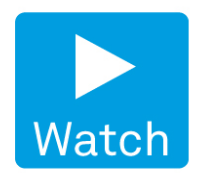




\title{
Large negative uniaxial magnetic anisotropy in highly distorted Co-ferrite thin films
}

\author{
Cite as: Appl. Phys. Lett. 114, 092408 (2019); doi: 10.1063/1.5064845 \\ Submitted: 9 October 2018 - Accepted: 7 February 2019 . \\ Published Online: 8 March 2019
}

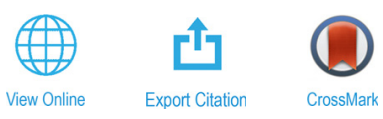

Takeshi Tainosho, ${ }^{7}$ Jun-ichiro Inoue, ${ }^{\top}$ Sonia Sharmin, ${ }^{\top}$ Masaki Takeguchi, ${ }^{2}$ Eiji Kita, ${ }^{1}$ and Hideto Yanagihara, ${ }^{1,3, a)}$ (D)

\begin{abstract}
AFFILIATIONS
'Department of Applied Physics, University of Tsukuba, 1-1-1 Tennodai, Tsukuba, Ibaraki 305-8573, Japan

${ }^{2}$ Transmission Electron Microscopy Station, National Institute for Materials Science (NIMS), 1-2-1 Sengen, Tsukuba 305-0047, Japan

${ }^{3}$ Tsukuba Research Center for Energy Materials Science (TREMS), University of Tsukuba, 1-1-1 Tennodai, Tsukuba, Ibaraki 305-8573, Japan
\end{abstract}

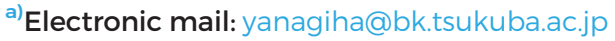

\begin{abstract}
The strain induced magnetic anisotropy (MA) of epitaxial Co-ferrite (CFO) thin films grown on $\mathrm{MgAl}_{2} \mathrm{O}_{4}(001)$ (MAO) by reactive sputtering was studied. These films underwent large tetragonal compressive strain due to the lattice mismatch between the substrates and films, resulting in tetragonalities of up to -0.04 . Scanning transmission electron microscopy observation combined with fast Fourier transform analysis revealed that the lattice distortion monotonically relaxed with the increasing film thickness. Unlike the $\mathrm{CFO}(001)$ films on $\mathrm{MgO}(001)$ substrates, a magnetically enhanced layer exists at the interface between CFO and MAO. A large negative uniaxial MA energy of $-5.9 \mathrm{MJ} / \mathrm{m}^{3}$ was confirmed for the thinnest film of $12.9 \mathrm{~nm}$ at $300 \mathrm{~K}$ by magneto-torque measurements. The induced uniaxial anisotropy decreased with the increasing film thickness owing to misfit relaxation. The magneto-elastic (ME) constant, which was determined by a fitting line for films with different thicknesses and therefore different tetragonalities, was $0.15 \pm 0.01 \mathrm{GJ} / \mathrm{m}^{3}$. This result is consistent with the value of $0.14 \mathrm{GJ} / \mathrm{m}^{3}$ for the bulk and suggests that the framework of the phenomenological ME theory for CFO is valid for a wide range of tetragonalities, at least up to -0.04 . Our results also indicate that the misfit engineering of CFO thin films has great potential in inducing enhanced uniaxial MA.
\end{abstract}

Published under license by AIP Publishing. https://doi.org/10.1063/1.5064845

A divalent Co ion located at an octahedral site possesses orbital angular momentum in a periodical crystal field. ${ }^{1}$ Because magnetic anisotropy (MA) mainly arises from spin-orbit interactions, most magnetic compounds containing $\mathrm{Co}^{2+}$ exhibit large MA. Among magnetic oxides containing $\mathrm{Co}^{2+}$ ions, Co-ferrite (CFO) belongs to the class of magnetic materials with the highest Néel temperature, and CFO structures with cubic symmetry have been shown to exhibit significantly large $\mathrm{MA}^{2}$ Because of the large orbital angular momentum of $\mathrm{Co}^{2+}, \mathrm{CFO}$ also exhibits considerably large magneto-elastic (ME) coupling. ${ }^{3}$ In this sense, $\mathrm{CFO}$ is a good candidate for demonstrating large induced MA through the ME effect. ${ }^{4,5}$ In general, the ME effect can be understood within the framework of the phenomenological model, where the induced MA energy is simply proportional to the applied strain. However, it is not clear how much anisotropy energy can be induced by applying strain and/or what is the maximum valid limit of distortion.

To introduce large uniaxial strain into thin films, heteroepitaxy is a promising technique. ${ }^{6,7}$ The induced MA energy through the ME effect is calculated using a stress tensor, elastic constants, and the magnetostriction constants of the material. According to the conventional phenomenological theory, uniaxial MA energy $\left(K_{\mathrm{u}}\right)$ can be expressed as a simple linear function of tetragonality $\varepsilon_{1}-\varepsilon_{2}$. Here, $\varepsilon_{1}=\left(a_{\|}\right.$ $\left.-a_{0}\right) / a_{0}$ and $\varepsilon_{2}=\left(a_{\perp}-a_{0}\right) / a_{0}$ indicate the in-plane and out-ofplane distortions, where $a_{\|}, a_{\perp}$, and $a_{0}$ are the distorted in-plane and out-of-plane lattice constants and the intrinsic bulk lattice constant, respectively. For cubic systems with a tetragonal distortion, the induced $K_{\mathrm{u}}$ is given $K_{\mathrm{u}}=\frac{3}{2} \lambda_{100}\left(C_{12}-C_{11}\right)\left(\varepsilon_{1}-\varepsilon_{2}\right) .{ }^{8}$ Here, $\lambda_{100}$, $C_{11}$, and $C_{12}$ are the magnetostriction constant and elastic moduli, respectively. The term $\frac{3}{2} \lambda_{100}\left(C_{12}-C_{11}\right)$ is called the ME constant $B_{1}$ and hence $K_{\mathrm{u}}=B_{1}\left(\varepsilon_{1}-\varepsilon_{2}\right)$.

CFO thin films deposited on various substrates have previously been investigated considering the various effects of lattice distortion inducing MA via the ME effect. ${ }^{9-11}$ Unfortunately, the saturation magnetization value is usually far below the bulk value of $425 \mathrm{kA} / \mathrm{m}$, which corresponds to an inverse cation distribution in the spinel structure with a significant orbital moment of approximately $0.6 \mu_{\mathrm{B}}$ that is 
associated with the octahedrally coordinated $\mathrm{Co}^{2+}$ ions. ${ }^{2}$ However, Niizeki et al. recently developed a technique to grow high-quality CFO thin films on $\mathrm{MgO}(001)$ by reactive $\mathrm{RF}$ magnetron sputtering. ${ }^{12}$ The CFO epitaxial films exhibited saturation magnetization $M_{\mathrm{s}}=430 \mathrm{kA} / \mathrm{m}$ comparable to that of the bulk and an induced $K_{\mathrm{u}}$ close to the value of $1.4 \mathrm{MJ} / \mathrm{m}^{3}$, as expected from the phenomenological ME theory for epitaxially strained $\mathrm{CFO}$ on $\mathrm{MgO}(001)$. Thus, the $\mathrm{ME}$ model in the $\mathrm{CFO}(001)$ epitaxial system for small distortion $\left(\varepsilon_{1}-\varepsilon_{2}=0.015\right)$ was validated using an appropriate growth technique.

In this study, we experimentally investigated the ME coupling of CFO films grown on $\mathrm{MgAl}_{2} \mathrm{O}_{4}(001)$ (MAO) substrates by reactive $\mathrm{RF}$ magnetron sputtering. The lattice constants of $\mathrm{CFO}$ and MAO are $0.838 \mathrm{~nm}$ and $0.808 \mathrm{~nm}$, respectively; therefore, relatively large tetragonal compressive strain can be introduced. We confirmed the largest value of tetragonality to be $\varepsilon_{1}-\varepsilon_{2}=-0.04$. The thinnest film showed an extremely large negative $K_{\mathrm{u}}$ of $-5.9 \mathrm{MJ} / \mathrm{m}^{3}$. The strain induced MA energy was found to be consistent with that expected from the phenomenological ME theory.

The details of our experiments are as follows. Polished $\mathrm{MAO}(001)$ substrates were cleaned with acetone and ethanol in an ultrasonic bath for $5 \mathrm{~min}$ and annealed for $1 \mathrm{~h}$ at $773 \mathrm{~K}$ in a vacuum chamber, ${ }^{12}$ where the base pressure was $5 \times 10^{-6} \mathrm{~Pa}$. The FeCo (3:1 atom ratio) alloy was used as a sputtering target. $\mathrm{Ar}$ and $\mathrm{O}_{2}$ gases were flown as process gases at rates of $30.0 \mathrm{sccm}(\sim 0.5 \mathrm{~Pa})$ and $6.0 \mathrm{sccm}(\sim 0.1 \mathrm{~Pa})$, respectively. The substrate temperature was maintained at $773 \mathrm{~K}$ during the deposition. To change the amount of strain, several CFO thin films of different thicknesses ranging from 12.9 to $81.6 \mathrm{~nm}$ were prepared. The surface structure was observed by reflection high-energy electron diffraction (RHEED). The thicknesses were evaluated by X-ray reflectivity (Rigaku, MiniFlex600). To determine the in-plane and out-of-plane lattice constants, X-ray diffraction (XRD) was performed (Rigaku, SmartLab, and MiniFlex600). The microstructure around the interface was observed by scanning transmission electron microscopy (STEM) (JEOL, JEMARM200). In-plane and out-of-plane magnetization curves were measured using a SQUID vibrating sample magnetometer (Quantum Design, MPMS3) at $300 \mathrm{~K}$. To determine the induced MA, magnetic torque measurements were performed at $300 \mathrm{~K}$ (Quantum Design, PPMS).

Typical RHEED patterns of MAO and CFO into [100] azimuth are presented in Figs. 1(a) and 1(b), respectively, indicating epitaxial growth of the CFO thin film. Typical streak patterns of a spinel structure are observed, and a reconstruction of the surface structure is confirmed, as in the case of epitaxial $\mathrm{Fe}_{3} \mathrm{O}_{4}(001)$ thin films. ${ }^{13,14}$

Next, in-plane and out-of-plane lattice constants $\left(\bar{a}_{\|}\right.$and $\left.\bar{a}_{\perp}\right)$ of the films were evaluated from the corresponding XRD measurements, as shown in Figs. 1 (c) and $1(\mathrm{~d})$, respectively. Both $\bar{a}_{\|}$and $\bar{a}_{\perp}$ are dependent on the film thickness, as shown in Fig. 1(e). $\bar{a}_{\|}$and $\bar{a}_{\perp}$ become close to $a_{0}$ when increasing the film thickness, signifying that misfit relaxation occurs at least with those thicknesses. Therefore, the observed $\bar{a}_{\|}$and $\bar{a}_{\perp}$ are both volume average lattice constants. Figure 1(f) shows $\varepsilon_{2}$ vs $-\varepsilon_{1}$ plots, where the slope of the fitting line provides the apparent Poisson's ratio ${ }^{15}\left(-\varepsilon_{2} / \varepsilon_{1}=2 \nu /(1-\nu)\right)$ of $1.06 \pm 0.02$, indicating that the Poisson's ratio of the $\mathrm{CFO}(001)$ film is $\nu=0.35 \pm 0.01$. Note that this value is close to that reported by Lisfi et al. ${ }^{16}$ and slightly smaller than that reported by Niizeki et al. ${ }^{12}$

To investigate the interfacial structure, including the misfit relaxation, we carefully investigated the cross sectional STEM image of the $\mathrm{CFO}(81.6 \mathrm{~nm}) / \mathrm{MAO}(001)$ film shown in Fig. 2(a). Figures 2(b)-2(d)
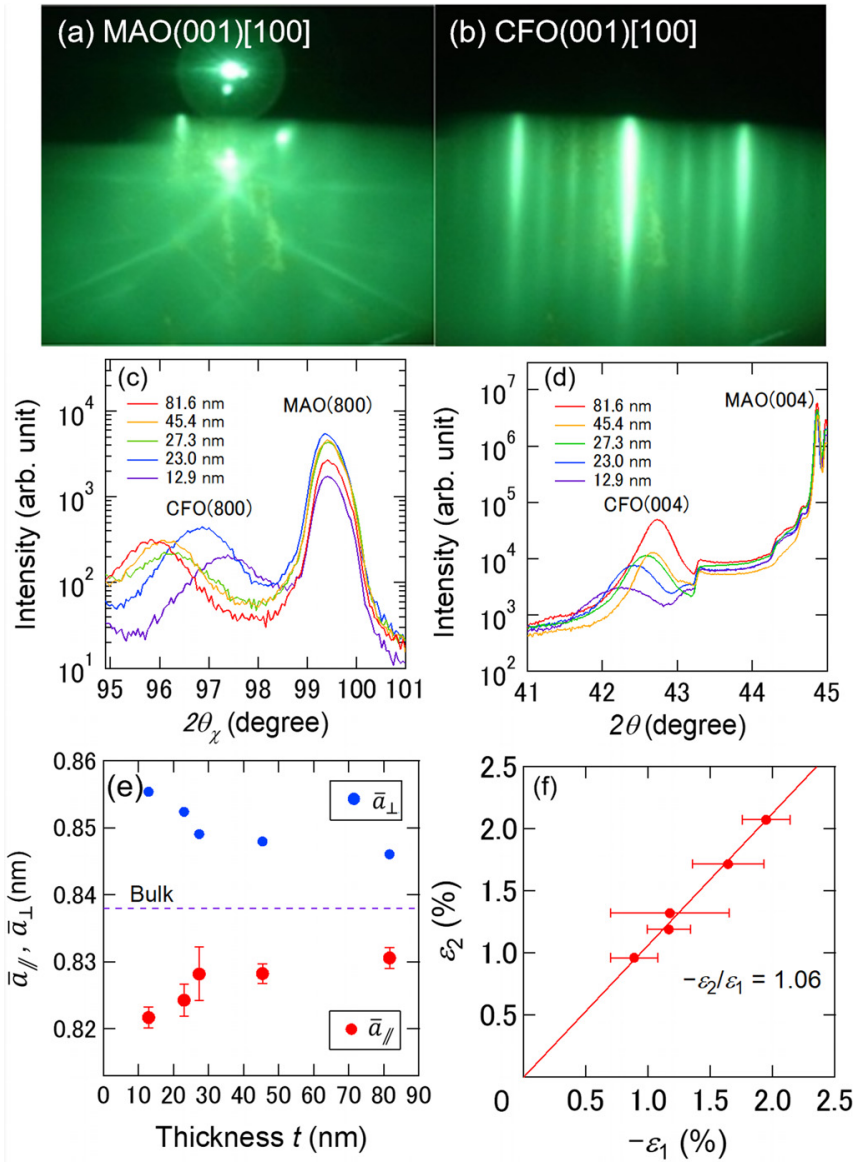

FIG. 1. RHEED patterns obtained in [100] azimuth of (a) a $M A O(001)$ substrate and (b) a CFO(001) thin film. (c) and (d): In-plane and out-of-plane XRD patterns. The peak shift depending on the thickness indicates that a thinner film is more distorted. (e) The thickness dependence of $\bar{a}_{\|}$and $\bar{a}_{\perp}$. (f) A plot of $-\varepsilon_{1}$ vs $\varepsilon_{2}$. The slope of the fitting line indicates an apparent Poisson ratio of $1.06 \pm 0.02$.

show the fast Fourier transform (FFT) image converted from Fig. 2(a), the magnified STEM image near the interface, and the FFT filtering image corresponding to Fig. 2(c), respectively. Using the spacing of the $\mathrm{MAO}(001)$ substrate as a standard in Fig. 2(d), the mean lattice spacing of $\mathrm{CFO}(001)$ along the [100] direction $a_{\|}(D)$ was determined at a distance $D$ from the $\mathrm{MAO} / \mathrm{CFO}$ interface. For comparison with $\bar{a}_{\|}$ determined by XRD measurements, we derived the average lattice constant $\bar{a}_{\|}^{\text {STEM }}(D)=\frac{1}{D} \int_{0}^{D} a_{\|}(D) d D$, as shown in Fig. $2(e) \cdot \bar{a}_{\|}^{\text {STEM }}(D)$ and $\bar{a}_{\|}$were roughly consistent within the experimental error. One can see that misfit relaxation occurs at a very early stage in film growth. According to Gatel's report, ${ }^{17} \mathrm{CFO}(5 \mathrm{~nm}) / \mathrm{MAO}(001)$ films deposited by molecular beam epitaxy seem to grow coherently without misfit dislocation. This is inconsistent with our result, suggesting that film deposition methods affect the details of the growth process as well as the local structures. Moreover, in the case of $\mathrm{CFO} / \mathrm{MgO}(001)$ films grown by reactive magnetron sputtering, coherent growth was confirmed up to the $50 \mathrm{~nm}$ thick films. ${ }^{12}$ Thus, the misfit relaxation process of $\mathrm{CFO}(001)$ epitaxial films depends on the value of lattice mismatch, as well as its sign. 

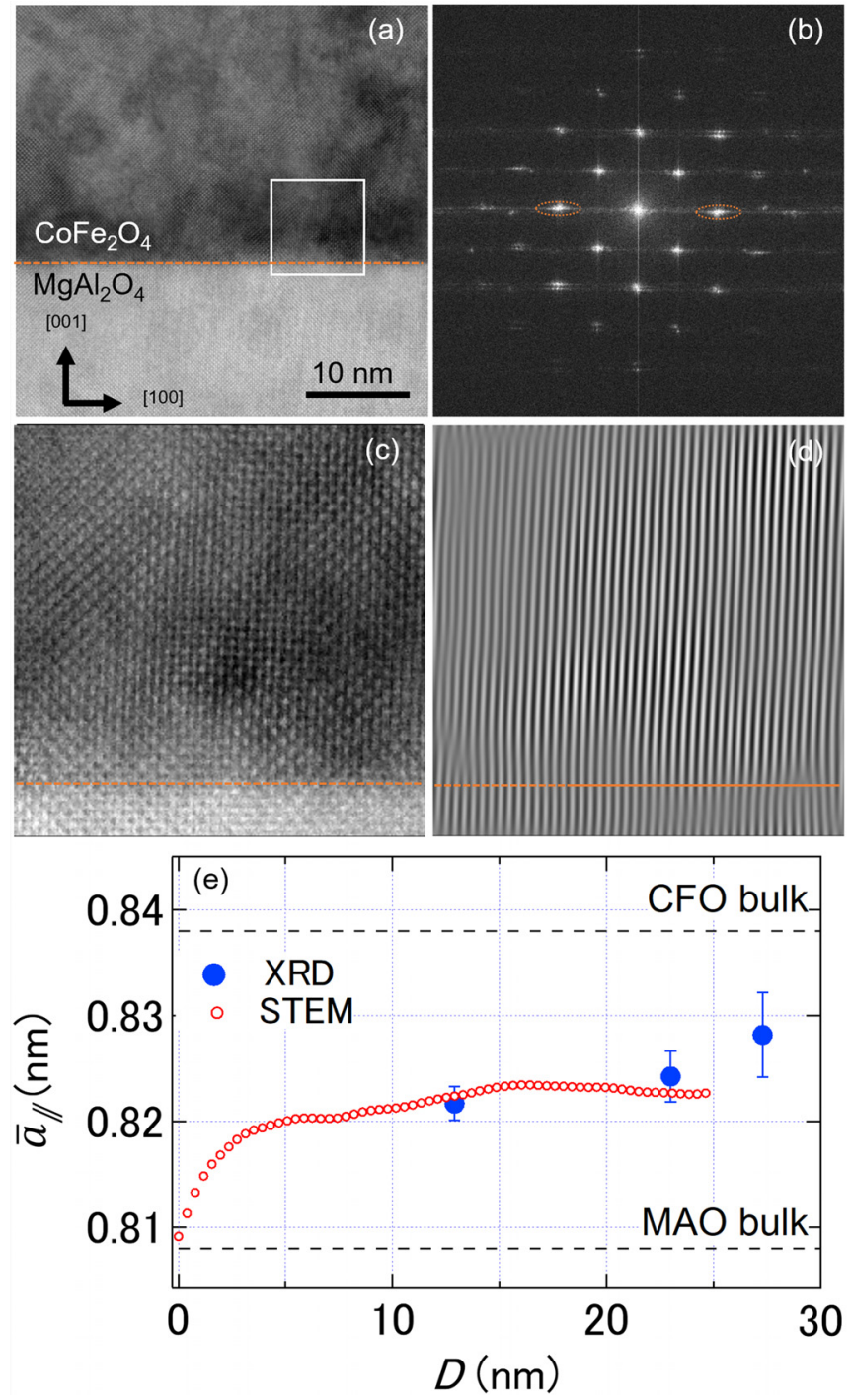

FIG. 2. (a) Cross sectional STEM image. (b) A FFT image of (a). (c) The magnified STEM image corresponding to the square region in (a). (d) The FFT filtering image of (a) where the mask is indicated as two ellipsoids in (b). Here, the region corresponding to (c) is shown. Horizontal dotted lines in (a), (c), and (d) indicate the interface. (e) The average lattice constant from the interface distance $D$ extracted from the FFT filtering image is shown as red circles. The blue markers show that evaluated by XRD.

Figure 3(a) shows the in-plane $M H$-curves of the films with different thicknesses. All films showed $M_{\mathrm{s}}$ comparable to the bulk $\left(M_{\mathrm{s}}\right.$ $=425 \mathrm{kA} / \mathrm{m}$ ). Figures $3(\mathrm{~b})$ and $3(\mathrm{c})$ show the thickness dependence of $M_{\mathrm{s}}$ and areal saturation magnetization $M_{\mathrm{s}} \cdot t$, respectively. In the case of $\mathrm{CFO}$ films on $\mathrm{MgO}(001)$ substrates, the thickness of a magnetic dead layer has been reported to be $2-5 \mathrm{~nm} .{ }^{12,18}$ The dead layer was believed to originate from high-density anti-phase boundaries (APBs) because of the difference in crystal structures between $\mathrm{MgO}$ and CFO. On the other hand, in the case of $\mathrm{CFO} / \mathrm{MAO}(001)$, the intercept of the vertical axis was positive which is $0.76 \pm 0.16 \times 10^{-3} \mathrm{~A}$, which
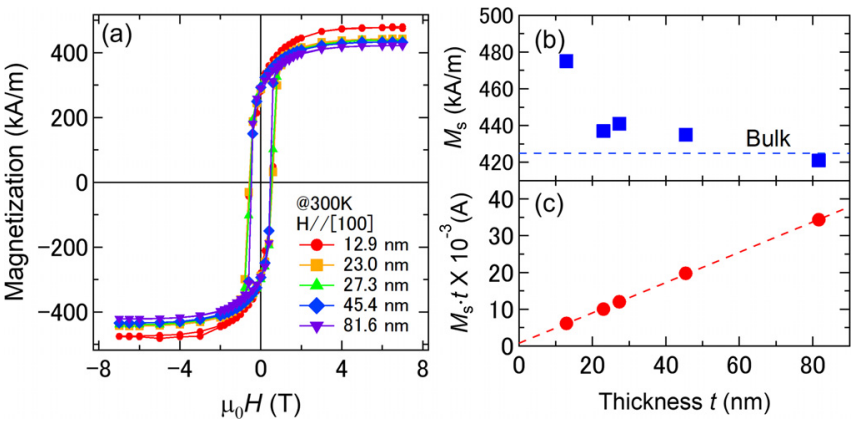

FIG. 3. (a) The $M H$-curves of all the samples with different thicknesses. (b) Plot of $M_{\mathrm{s}}$ and (c) thickness dependences of $M_{\mathrm{s}} \cdot t$. The intercept and slope in (c) are $0.76 \pm 0.16 \times 10^{-3} \mathrm{~A}$ and $412 \pm 4 \mathrm{kA} / \mathrm{m}$, respectively.

implies that instead of a dead layer, there is a part enhancing magnetization around the interface. Because both $\mathrm{MAO}$ and $\mathrm{CFO}$ are isostructural, MAO is an appropriate material to reduce interfacial defects such as APBs. ${ }^{19,20}$ The layer contributing to increased magnetization may originate from charge discontinuity at the interface between normal spinel MAO and inverse spinel. ${ }^{21}$ Another possible explanation for the increase in magnetization is the orbital moment of $\mathrm{Co}^{2+}$ at the vicinity of the interface caused by the large lattice distortion. Note that the saturation fields for the out-of-plane $M H$-curves are higher than $\mu_{0} H=7 \mathrm{~T}$, and we therefore neither obtain plausible $M H$-curves nor determine $K_{\mathrm{u}}$ by taking into account the difference between the inplane and out-of-plane $\mathrm{MH}$-curves. We note that the error bars in Fig. 3 (c) are smaller than the size of markers.

To evaluate $K_{\mathrm{u}}$ quantitatively, angle dependent magneto-torque $L(\theta)$ was measured. Here, $\theta$ stands for the angle between the external field and the [001] direction of the film. The field was rotated in the (010) plane. Figure 4(a) shows the magneto-torque curves of CFO $(45.4 \mathrm{~nm}) / \mathrm{MAO}(001)$ at various fields up to $\mu_{0} H=9 \mathrm{~T}$. Since the anisotropy energy with a uniaxial symmetry is roughly expressed as $E_{\mathrm{A}}=K_{\mathrm{u}} \sin ^{2} \varphi$, where $\varphi$ stands for an angle between the magnetization direction and the direction normal to the film plane, the shape of $L(\theta)$ becomes sinusoidal if the magnetization direction coincides with
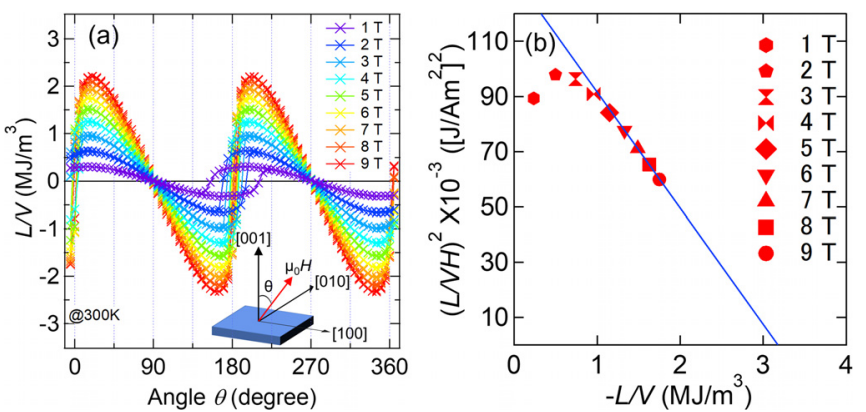

FIG. 4. (a) Magneto-torque curves of $\mathrm{CFO}(001)(45 \mathrm{~nm}) / \mathrm{MAO}$. The saw-tooth-like curves represent the large magnetic anisotropy field. The inset shows the experimental geometry. (b) Miyajima's plot, where the linear fitting was performed for over $6 \mathrm{~T}$, as shown by the blue line. Here, we exclude the torque data at the lower field due to multi-domain states. The error bars are less than the size of the markers. The $x$-intercept represents the effective $K_{u}=-3.2 \mathrm{MJ} / \mathrm{m}^{3}$. 
that of the external field. However, the values of $L(\theta)$ when plotted show saw-tooth-like curves with rotational hysteresis, which indicate that the anisotropy field is much greater than $\mu_{0} H=9 \mathrm{~T}$. One can find the angles of $L(\theta)=0$ at $\theta \approx 0^{\circ}\left(180^{\circ}\right)$ and $\theta \approx 90^{\circ}\left(270^{\circ}\right)$. As the slope at $\theta \approx 0^{\circ}\left(180^{\circ}\right)$ is positive and steep whereas that at $\theta$ $\approx 90^{\circ}\left(270^{\circ}\right)$ is negative and gentle, $\theta \approx 0^{\circ}\left(180^{\circ}\right)$ corresponds to the hard direction, and $\theta \approx 90^{\circ}\left(270^{\circ}\right)$ corresponds to the easy direction. To obtain accurate $K_{\mathrm{u}}$, we adapted Miyajima's method as shown in Fig. 4(b). ${ }^{22}$ In this method, we plot the torque data taken at $\theta=45^{\circ}$ as $(L(\theta) / V H)^{2}$ versus $-L(\theta) / V$, where $V$ represents the sample volume. If the magnetic field is large enough to make a single domain, data points taken at different magnetic fields will be aligned as a straight line. The slope and horizontal intercept of the line give $M_{\mathrm{s}}$ and $K_{\mathrm{u}}$, respectively. We fitted the plots at $\mu_{0} H \geq 6 \mathrm{~T}$ as a linear function drawn by a blue line in Fig. 4(b) and obtained $M_{\mathrm{s}}$ and $K_{\mathrm{u}}$ to be $410 \mathrm{kA} / \mathrm{m}$ and $-3.2 \mathrm{MJ} / \mathrm{m}^{3}$, respectively. Since the shape anisotropy energy is $\mu_{0} M_{\mathrm{s}}^{2} / 2 \approx-0.1 \mathrm{MJ} / \mathrm{m}^{3}$, the intrinsic $K_{\mathrm{u}}$ induced by the magnetoelastic effect is $-3.1 \mathrm{MJ} / \mathrm{m}^{3}$. Note that the $M_{\mathrm{s}}$ extracted from the torque analysis was reasonably close to the value obtained from the $M H$-curve measurement $\left(M_{\mathrm{s}}=435 \mathrm{kA} / \mathrm{m}\right)$. In-plane torque curves were also measured to evaluate MA in the plane. Magneto-crystalline anisotropy energy $K_{1}$ with four-fold symmetry was estimated to be $0.2 \mathrm{MJ} / \mathrm{m}^{3}$ from the $4 \theta$ component (not shown), which is comparable to the bulk value of $0.29 \mathrm{MJ} / \mathrm{m}^{3}$.

Next, we discuss whether the induced $K_{\mathrm{u}}$ can be explained by the phenomenological ME theory. Figure 5(a) shows the tetragonality dependence of the induced $K_{\mathrm{u}}$. The best fit red line expresses a linear relationship with the slope corresponding to a ME constant $B_{1}=0.15 \pm 0.01 \mathrm{GJ} / \mathrm{m}^{3}$. This value is almost the same as $0.14 \mathrm{GJ} / \mathrm{m}^{3}$, estimated from bulk parameters $\left(B_{1}=\frac{3}{2} \lambda_{100}\left(C_{12}-C_{11}\right)\right.$, where $\lambda_{100}$ $=-590 \times 10^{-6}, C_{11}=273 \mathrm{GPa}$, and $\left.C_{12}=106 \mathrm{GPa}\right) .{ }^{3,9}$ This supports the validity of the phenomenological model of $K_{\mathrm{u}}=B_{1}\left(\varepsilon_{1}-\varepsilon_{2}\right)$ at least up to $\varepsilon_{1}-\varepsilon_{2}=-0.04$.

Inoue et al. recently calculated the induced $\mathrm{MA}$ of a $\mathrm{Co}^{2+}$ ion located in octahedrally coordinated B-site of a spinel ferrite within a framework of the cluster model by the tight binding method. ${ }^{23}$ In their calculation, they incorporated the local crystal field of $V_{\mathrm{E}}$ instead the tetragonality $\varepsilon_{1}-\varepsilon_{2}$. The induced $K_{\mathrm{u}}$ dependence on $V_{\mathrm{E}}$ in the CFO
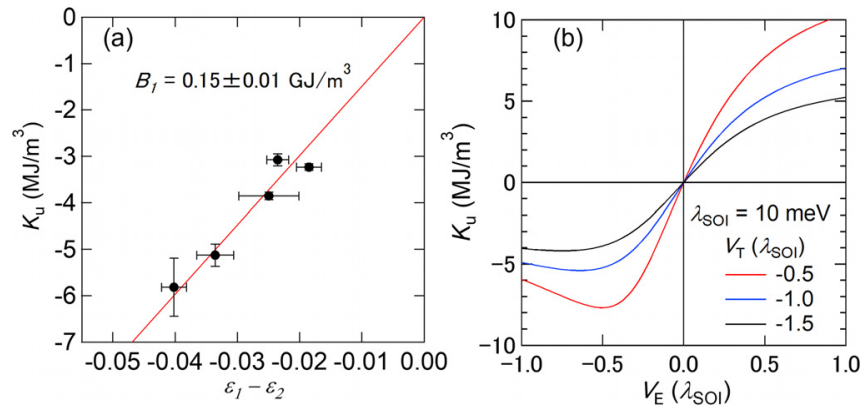

FIG. 5. (a) Experimentally obtained $K_{\mathrm{u}}$ vs tetragonality plot. The red line is the fitting result as a linear function, whose slope indicates $B_{1}=0.15 \pm 0.01 \mathrm{GJ} / \mathrm{m}^{3}$. (b) Calculation result of $K_{u}$ vs $V_{E}$ with three types of $V_{T}$ redrawn from Ref. 23, assuming $\lambda_{\mathrm{SOI}}=10 \mathrm{meV}$. In this calculation, the tetragonality is expressed as a strength of the tetragonal crystal field and measured in the spin-orbit constant $\lambda_{\mathrm{sol}}$ of $\mathrm{Co}^{2+}$. Reproduced with permission from Inoue et al. Mater. Res. Express. 1, 046106 (2014), Copyright 2014 IOP Publishing Ltd. cluster was well explained over a wide range, and the valid range of the phenomenological relationship of $K_{\mathrm{u}}=B_{1}\left(\varepsilon_{1}-\varepsilon_{2}\right)$ as well as the $K_{\mathrm{u}}$ behavior at both limits of the tetragonal distortions was discussed. ${ }^{23,24}$ Because of the difficult treatment of electron correlation, to calculate $K_{\mathrm{u}}$, they extended the single ion model to a cluster composed of nearest neighbor oxygen ions and the next nearest neighbor cations. In addition, their calculations were performed within the framework of a semi-quantitative measure of the tetragonal distortion, thus signifying that their calculation results contained some relative parameters.

As shown in Fig. 5(b), this cluster calculation ${ }^{23}$ predicts the following characteristic behaviors in a tetragonally distorted $\mathrm{CFO}(001)$ film. (i) When the tetragonal lattice distortion is small enough, the induced $K_{\mathrm{u}}$ is proportional to the tetragonality, as described in the phenomenological ME theory. (ii) The linearly changing region of $K_{\mathrm{u}}$ is almost symmetric to the origin. (iii) With increasing $\varepsilon_{1}-\varepsilon_{2}, K_{\mathrm{u}}$ reaches the order of the magnitude of the spin-orbit interaction $\lambda_{\text {SOI }}$ of $\mathrm{Co}^{2+}$. (iv) On the contrary, when $\varepsilon_{1}-\varepsilon_{2}$ decreases as a result of the application of compressive stress, $K_{\mathrm{u}}$ exhibits a dip and then approaches zero. Using Ref. 23, we replotted $K_{\mathrm{u}}$ in terms of $\mathrm{MJ} / \mathrm{m}^{3}$ for $\lambda_{\mathrm{SOI}}=10 \mathrm{meV}^{24}$ By assuming a trigonal crystal field potential of $V_{\mathrm{T}}$ $=-0.5 \lambda_{\mathrm{SOI}}$ and a tetragonal crystal field potential of $V_{\mathrm{E}}=-0.5 \lambda_{\mathrm{SOI}}$, the minimum $K_{\mathrm{u}}$ expected from the cluster model calculation was approximately $-8 \mathrm{MJ} / \mathrm{m}^{3}$. This implies that a more negative $K_{\mathrm{u}}$ can be induced in $\mathrm{CFO}(001)$ films if larger compressive stresses are applied. We note that such a large MA and high electrical resistivity of CFO are the potential characteristics for high frequency devices. ${ }^{25}$ The anisotropy fields for magnetization rotations in out-of-plane and inplane are $H_{a 1}=21.5 \mathrm{MA} / \mathrm{m}$ and $H_{a 2}=0.75 \mathrm{MA} / \mathrm{m}$, respectively. The resonance frequency ${ }^{26} f_{\mathrm{r}}=\nu_{0} / 2 \pi \sqrt{H_{a 1} H_{a 2}}$ can thus reach approximately $140 \mathrm{GHz}$. Here, $\nu_{0}$ is the gyromagnetic ratio.

As mentioned above, the observed $K_{\mathrm{u}}$ of $\mathrm{CFO} / \mathrm{MAO}(001)$ with $\varepsilon_{1}-\varepsilon_{2}=-0.04$ can be explained by phenomenological ME theory of $K_{\mathrm{u}}=B_{1}\left(\varepsilon_{1}-\varepsilon_{2}\right)$. Moreover, the cluster model calculation suggests that the induced $K_{\mathrm{u}}$ is symmetric in the valid range of the phenomenological model. Therefore, if a $\mathrm{CFO}(001)$ film with $\varepsilon_{1}-\varepsilon_{2}=0.04$ is grown, a positive $K_{\mathrm{u}}$ as large as $6 \mathrm{MJ} / \mathrm{m}^{3}$ may be expected, which is greater than that of $\mathrm{Nd}_{2} \mathrm{Fe}_{14} \mathrm{~B}$.

In summary, we have examined the validity of the magnetoelastic theory for $\mathrm{CFO}(001)$ epitaxial films grown on $\mathrm{MAO}(001)$ substrates. The CFO thin films were deposited by reactive RF magnetron sputtering, and all samples showed saturation magnetization comparable to the bulk one. In the thinnest film, a large compressive strain $\left(\varepsilon_{1}-\varepsilon_{2}=-0.04\right)$ was confirmed by XRD measurements, and large negative $K_{\mathrm{u}}=-5.9 \mathrm{MJ} / \mathrm{m}^{3}$ was confirmed by magneto-torque measurements combined with the extrapolation analysis technique. The estimated value of magneto-elastic coefficient $B_{1}$ was $0.15 \pm 0.01 \mathrm{GJ} /$ $\mathrm{m}^{3}$, which was consistent with the bulk value of $0.14 \mathrm{GJ} / \mathrm{m}^{3}$. Although the lattice misfit between the $\mathrm{CFO}(001)$ and $\mathrm{MAO}(001)$ is as large as $-3.58 \%$, the strain-induced MA energy for the compressive tetragonal distortion can be quantitatively explained by the magneto-elastic model. Our results suggest the potential of distorted CFO for high frequency devices in the millimeter band.

This work was supported by the Japan Science and Technology Agency (JST) under Collaborative Research Based on Industrial Demand "High Performance Magnets: Towards Innovative Development of Next Generation Magnets." A part of this work was supported by NIMS microstructural characterization platform as a 
program of "Nanotechnology Platform" of the Ministry of Education, Culture, Sports, Science, and Technology (MEXT), Japan.

\section{REFERENCES}

${ }^{1}$ G. H. O. Daalderop, P. J. Kelly, and M. F. H. Schuurmans, Phys. Rev. B 44, 12054 (1991).

2J. C. Slonczewski, Phys. Rev. 110, 1341 (1958).

${ }^{3}$ R. M. Bozorth, E. F. Tilden, and A. J. Williams, Phys. Rev. 99, 1788 (1955).

${ }^{4}$ Y. Suzuki, G. Hu, R. B. B. van Dover, and R. J. J. Cava, J. Magn. Magn. Mater. 191, 1 (1999).

${ }^{\mathbf{5}}$ N. Somaiah, T. V. Jayaraman, P. A. Joy, and D. Das, J. Magn. Magn. Mater. 324, 2286 (2012).

${ }^{6}$ M. L. Lee, E. A. Fitzgerald, M. T. Bulsara, M. T. Currie, and A. Lochtefeld, J. Appl. Phys. 97, 011101 (2005).

${ }^{7}$ C. L. Canedy, H. Li, S. P. Alpay, L. Salamanca-Riba, A. L. Roytburd, and R. Ramesh, Appl. Phys. Lett. 77, 1695 (2000).

${ }^{8}$ B. Schulz and K. Baberschke, Phys. Rev. B 50, 13467 (1994).

${ }^{9}$ G. Hu, J. H. Choi, C. B. Eom, V. G. Harris, and Y. Suzuki, Phys. Rev. B 62, 779 (2000).

${ }^{10}$ F. Eskandari, S. B. Porter, M. Venkatesan, P. Kameli, K. Rode, and J. M. D. Coey, Phys. Rev. Mater. 1, 074413 (2017).

${ }^{11}$ H. Yanagihara, K. Uwabo, M. Minagawa, E. Kita, and N. Hirota, J. Appl. Phys. 109, 07C122 (2011).

${ }^{12}$ T. Niizeki, Y. Utsumi, R. Aoyama, H. Yanagihara, J. Inoue, Y. Yamasaki, H. Nakao, K. Koike, and E. Kita, Appl. Phys. Lett. 103, 162407 (2013).

${ }^{13}$ S. A. Chambers and S. A. Joyce, Surf. Sci. 420, 111 (1999).
${ }^{14}$ C. F. Chang, Z. Hu, S. Klein, X. H. Liu, R. Sutarto, A. Tanaka, J. C. Cezar, N. B. Brookes, H.-J. Lin, H. H. Hsieh, C. T. Chen, A. D. Rata, and L. H. Tjeng, Phys. Rev. X 6, 041011 (2016).

${ }^{15}$ M. Foerster, M. Iliev, N. Dix, X. Martí, M. Barchuk, F. Sánchez, and J. Fontcuberta, Adv. Funct. Mater. 22, 4344 (2012).

${ }^{16}$ A. Lisfi, C. M. Williams, L. T. Nguyen, J. C. Lodder, A. Coleman, H. Corcoran, A. Johnson, P. Chang, A. Kumar, and W. Morgan, Phys. Rev. B 76, 054405 (2007).

${ }^{17}$ C. Gatel, B. Warot-Fonrose, S. Matzen, and J. B. Moussy, Appl. Phys. Lett. 103, 092405 (2013).

${ }^{18}$ H. Yanagihara, Y. Utsumi, T. Niizeki, J. Inoue, and E. Kita, J. Appl. Phys. 115, 17A719 (2014).

${ }^{19}$ W. Eerenstein, T. T. M. Palstra, T. Hibma, and S. Celotto, Phys. Rev. B 66, 201101 (2002).

${ }^{20}$ W. Eerenstein, L. Kalev, L. Niesen, T. T. M. Palstra, and T. Hibma, J. Magn. Magn. Mater. 258-259, 73 (2003).

${ }^{21}$ M. Matsumoto, S. Sharmin, J. Inoue, E. Kita, and H. Yanagihara, IEEE Trans. Magn. 53, 2503604 (2017).

${ }^{22}$ H. Miyajima, K. Sato, and T. Mizoguchi, J. Appl. Phys. 47, 4669 (1976).

${ }^{23}$ J. Inoue, H. Yanagihara, and E. Kita, Mater. Res. Express 1, 046106 (2014).

${ }^{24}$ J. Inoue, T. Niizeki, H. Yanagihara, H. Itoh, and E. Kita, AIP Adv. 4, 02711 (2014).

${ }^{25}$ X. Zhang, S. Meng, D. Song, Y. Zhang, Z. Yue, and V. G. Harris, Sci. Rep. 7, 44193 (2017).

${ }^{26}$ C. Kittel, Phys. Rev. 73, 155 (1948).

${ }^{27}$ M. Sagawa, S. Fujimura, H. Yamamoto, Y. Matsuura, and S. Hirosawa, Jpn. J. Appl. Phys. Part 1 57, 4094 (1985). 